\title{
Effect of covalent bonding on magnetism and the missing neutron intensity in copper oxide compounds
}

\author{
Andrew C. Walters ${ }^{1,2,3}$, Toby G. Perring ${ }^{1,2}$, Jean-Sébastien Caux ${ }^{4}$, Andrei T. Savici ${ }^{5}$, Genda D. Gu ${ }^{5}$, \\ Chi-Cheng Lee ${ }^{5}$, Wei Ku ${ }^{5}$ and Igor A. Zaliznyak ${ }^{5 \star}$
}

\begin{abstract}
Theories involving highly energetic spin fluctuations are among the leading contenders for explaining high-temperature superconductivity in the cuprates ${ }^{1}$. These theories could be tested by inelastic neutron scattering (INS), as a change in the magnetic scattering intensity that marks the entry into the superconducting state provides a precise quantitative measure of the spin-interaction energy involved in the superconductivity ${ }^{2-11}$. However, the absolute intensities of spin fluctuations measured in neutron scattering experiments vary widely, and are usually much smaller than expected from fundamental sum rules, resulting in 'missing' INS intensity 2-5,12,13. Here, we solve this problem by studying magnetic excitations in the one-dimensional related compound, $\mathrm{Sr}_{2} \mathrm{CuO}_{3}$, for which an exact theory of the dynamical spin response has recently been developed. In this case, the missing INS intensity can be unambiguously identified and associated with the strongly covalent nature of magnetic orbitals. We find that whereas the energies of spin excitations in $\mathrm{Sr}_{2} \mathrm{CuO}_{3}$ are well described by the nearestneighbour spin-1/2 Heisenberg Hamiltonian, the corresponding magnetic INS intensities are modified markedly by the strong $2 p-3 d$ hybridization of $\mathrm{Cu}$ and $\mathrm{O}$ states. Hence, the ionic picture of magnetism, where spins reside on the atomic-like $3 d$ orbitals of $\mathrm{Cu}^{2+}$ ions, fails markedly in the cuprates.
\end{abstract}

Over the past 20 years, the magnetic properties of cuprates have been studied extensively by theorists and experimentalists alike. These systems are usually described within the antiferromagnetic Mott insulator model, in which the unpaired electrons are localized on the $\mathrm{Cu}^{2+}$ ions because of the overwhelming cost in the on-site Coulomb interaction energy, $U$, associated with the charge transfer between the $\mathrm{Cu}$ sites, a strong correlation phenomenon. Virtual electron hopping, which in the one-band Hubbard model of a Mott insulator often adopted for cuprates ${ }^{14}$ is quantified by the transition matrix element, $t$, results in antiferromagnetic exchange. For $t \ll U$, electronic spins form the only low-energy electronic degrees of freedom. Their properties are well approximated by the spin-1/2 Heisenberg Hamiltonian on the lattice ${ }^{15}, H=J \sum_{i(n n) j} \mathbf{S}_{i} \mathbf{S}_{j}$, with the nearest-neighbour exchange coupling $J \approx 4 t^{2} / U$.

This description conveniently splits the problem of electronic magnetism in the Mott insulator into two parts ${ }^{15}$. The first deals with the electron transfer between the neighbouring sites of the crystal lattice, which is determined by the overlap integral $(\sim t)$ of the wavefunctions occupied by the unpaired electrons, and leads to the Hubbard model, or the Heisenberg spin Hamiltonian. The second concerns the form of the electronic Wannier wavefunctions, that is, the shape of the spin magnetization cloud associated with each electron in the obtained Hubbard or Heisenberg model. It is determined by the crystal electric field and the hybridization of the valent $d$ orbitals with those of the surrounding $p$ orbitals of the anions - the covalency - and is usually addressed by firstprinciples calculations. The first part provides a description of the cooperative behaviour of electronic spins-spin order and spin excitations, whereas the second relates them to the behaviour of the magnetization density in the crystal, which interacts with external magnetic fields and is measured in actual experiments.

Although the importance of covalent bonding for the magnetism of transition-metal complexes was first noted by Pauling ${ }^{16}$ in 1931, transition-metal magnetic insulators are usually described within the ionic model, using the concepts of formal valence, ionic charge and charge transfer. The energy scale of covalent hybridization, which is responsible for spin superexchange and cooperative magnetism, is thus assumed to be a small perturbation of the ionic picture ${ }^{14,15}$. The hybridization effects then yield only small, $10-20 \%$ covalency corrections, and were traditionally considered unimportant for the magnetism in Mott insulators ${ }^{14,15}$. They were nevertheless noticed in the precise neutron diffraction study of $\mathrm{NiO}$ (ref. 17) and were elegantly explained by Hubbard and Marshall ${ }^{18}$. Modern polarized neutron diffraction experiments in systems with strongly correlated electrons also often find small magnetic moment density on the ligand site and the corresponding $\sim 10 \%$ covalent reduction of the magnetic moment at the $3 d$ site $^{19}$.

The picture differs markedly in the cuprates. Here, covalency has a fundamental role and could not be so easily discarded, which becomes clear when the state of the doped holes, leading to high-temperature superconductivity (HTSC), is considered. Zhang and Rice have shown ${ }^{20}$ that strong $\mathrm{Cu}(3 d)-\mathrm{O}(2 p)$ hybridization in fact defies Hund's rule and leads to an unusual singlet state of the doped hole, for which the wavefunction is mainly localized on oxygens, instead of the $S=1(3 d)$ (ref. 8) $\mathrm{Cu}^{3+}$ state, expected in the ionic picture. The corresponding extraordinary strength of $\mathrm{Cu}-\mathrm{O}$ covalent bonding is responsible for the recordhigh antiferromagnetic exchange couplings found in cuprates, $J \approx 1,500 \mathrm{~K}$ (refs 5,6) in the two-dimensional (2D) $\mathrm{La}_{2} \mathrm{CuO}_{4}$ and $J \approx 2,600 \mathrm{~K}$ (ref. 13) in its $1 \mathrm{D}$ chain relative $\mathrm{SrCuO}_{2}$. Such a distinctively dominant magnetic energy scale immediately suggests that it is a key player in the mechanisms of the HTSC. The close relationship between the magnetism and the HTSC is supported by a body of INS studies, in particular by discoveries of the sharp resonance peak of magnetic excitations and the low-energy incommensurate scattering, the temperature, doping

${ }^{1}$ ISIS Facility, Rutherford Appleton Laboratory, Chilton, Didcot OX11 OQX, UK, ${ }^{2}$ Department of Physics, University College London, Gower Street, London WC1E 6BT, UK, ${ }^{3}$ London Centre for Nanotechnology, 17-19 Gordon Street, London WC1H OAJ, UK, ${ }^{4}$ Institute for Theoretical Physics, University of Amsterdam, 1018 XE Amsterdam, The Netherlands, ${ }^{5}$ CMP\&MS Department, Brookhaven National Laboratory, Upton, New York 11973, USA. *e-mail:zaliznyak@bnl.gov. 
a
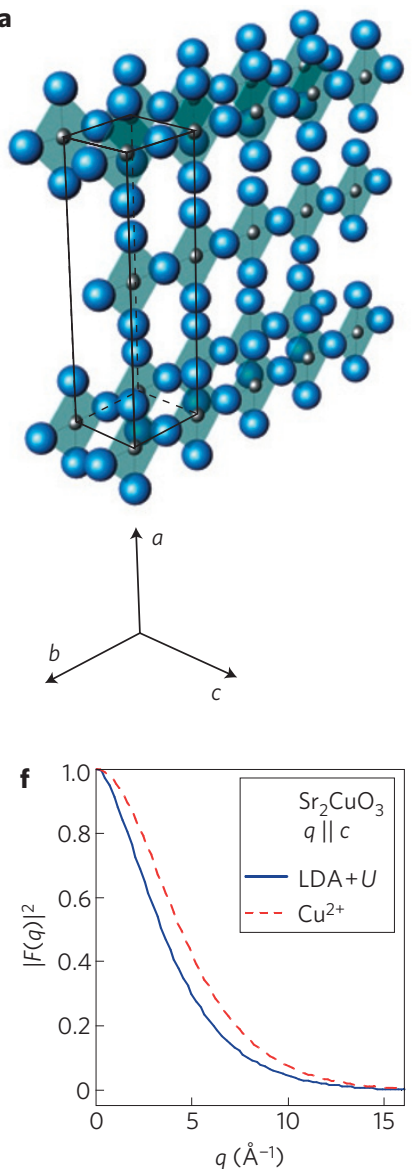

b

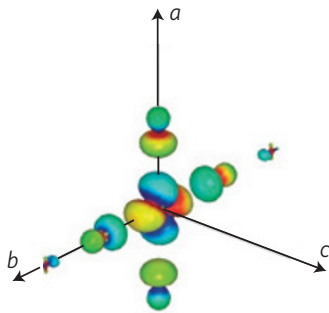

d

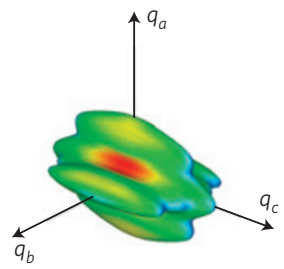

g

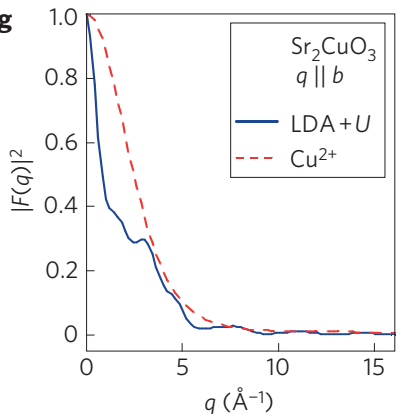

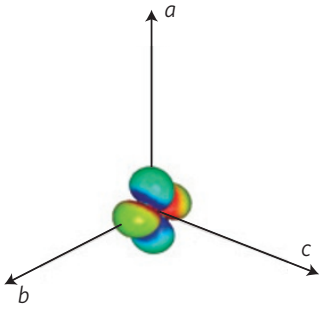

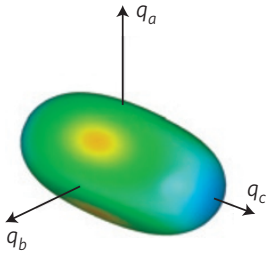

Figure 1 | Crystal structure and electronic orbitals in $\mathbf{S r}_{\mathbf{2}} \mathbf{C u O}_{\mathbf{3}} \cdot \mathbf{a}$, Corner-sharing $\mathrm{CuO}_{4}$ square plaquettes form chains along the $b$ axis. Spheres with half of the corresponding ionic radii show copper (small) and oxygen (large) ions. b, Covalently hybridized Wannier wavefunction of the unpaired magnetic electron in $\mathrm{Sr}_{2} \mathrm{CuO}_{3}$ obtained from the ab initio LDA $+U$ calculation. The equal-density surface at $|\psi(\mathbf{r})|^{2}=0.05 \AA^{-3}$ is shown. c, Similar depiction of the $\mathrm{Cu}^{2+}$ ionic wavefunction of the $3 d\left(x^{2}-y^{2}\right)$ orbital typically used for magnetic form factor calculations ${ }^{6,13,28,29}$. $\mathbf{d}, \mathbf{e}$, The equal-level surfaces of the magnetic form factor squared at $|F(\mathbf{Q})|^{2}=0.13 \approx 1 / e^{2}$ for the wavefunctions in $\mathbf{a}$ and $\mathbf{b}$, respectively. $\mathbf{f}-\mathbf{h}$, Comparison of the cuts of the magnetic form factors shown in $\mathbf{d}$ (solid blue line) and $\mathbf{e}$ (dashed red line) along the three symmetry directions.

and magnetic-field dependencies, all of which are correlated with superconductivity ${ }^{2,3,5}$.

Neutrons are an ideal probe of microscopic magnetism in condensed matter, as they interact with electronic magnetic moments directly, by means of the dipole-dipole force, and do not perturb charge distributions. The neutron magnetic scattering cross-section ${ }^{21}$ can be conveniently factored into the product of the dynamical spin-correlation function, $S(\mathbf{Q}, E)$, which is determined by the cooperative behaviour of electronic spins described by the Hubbard, $t-J$ or Heisenberg model, and the square of the magnetic form factor, $|F(\mathbf{Q})|^{2} . F(\mathbf{Q})$ is the Fourier transform of the electronic magnetization cloud associated with each spin, that is, its Wannier wavefunction, and includes the covalency effects. According to local density approximation (LDA) calculations ${ }^{22-24}$ and the general considerations outlined above, their impact on magnetic INS intensity could be extremely strong in cuprates.

Recent advances in neutron scattering technology have enabled magnetic excitations of energy up to $\sim 1 \mathrm{eV}$ to be measured routinely and with great precision. Previous measurements of the magnon dispersion in the HTSC parent $2 \mathrm{D}$ cuprate $\mathrm{La}_{2} \mathrm{CuO}_{4}$ were sufficiently sensitive to observe and quantify subtle deviations from the Heisenberg model, revealing the existence of ring exchange ${ }^{6}$. The analysis of the spectral weight of the spin-correlation function on the basis of the measured absolute INS magnetic intensity, however, has not been as successful. In fact, such analysis was typically carried out using the ionic $\mathrm{Cu}^{2+}$ magnetic form factor, which totally neglects the covalency effects. A number of studies ${ }^{5,6,13}$ have consequently found significant deviations of the INS spectral weight from sum rules, which in particular state that the integral of $S(\mathbf{Q}, E)$, over $\mathbf{Q}$ and energy should equal $N S(S+1)$, where $N$ is the number of magnetic sites in the sample and $S$ is the spin at each site ( $S=1 / 2$ for cuprates). Whereas covalency is a leading suspect for these outstanding discrepancies, their unambiguous association with the covalent magnetic form factor in planar cuprates is hindered by the absence of a precise theory describing the dynamical behaviour of the 2D spin system. Consequently, other explanations for the missing intensity have been developed, in particular, ascribing it to the salient features of $S(\mathbf{Q}, E)$ in the 2D Hubbard model ${ }^{12}$.

The situation is very different in $1 \mathrm{D}$, where recent progress in the theory of integrable models has led to the development of an extremely accurate quantitative theory for the dynamical spin correlations $^{25}$. Here, we report a detailed INS measurement of magnetic excitations in the $1 \mathrm{D}$ prototype cuprate material $\mathrm{Sr}_{2} \mathrm{CuO}_{3}$, the crystal structure of which is shown in Fig. 1a. It features chains of corner-sharing $\mathrm{CuO}_{4}$ plaquettes, which are also building blocks of 2D HTSC cuprate planes and related spin-ladders, running along the crystallographic $b$ axis. Although the direct determination of the in-chain spin exchange coupling is possible only by INS and is reported here, the estimate of $J \approx 250 \mathrm{meV}$ obtained from optical absorption measurements ${ }^{26}$ indicates a similar, or stronger degree of covalence as in $2 \mathrm{D}$ cuprates. The interchain orbital overlaps 
a

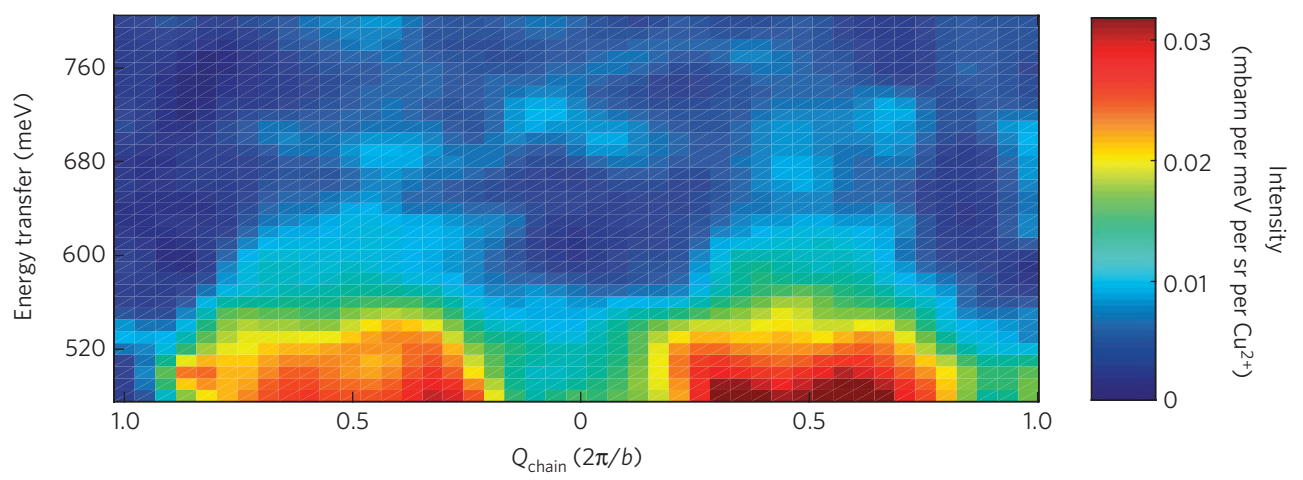

b

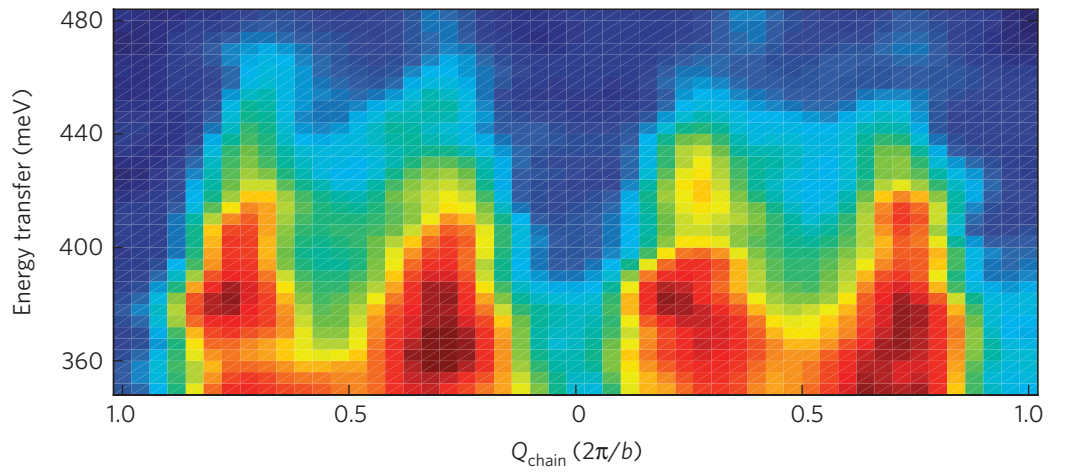

0.08
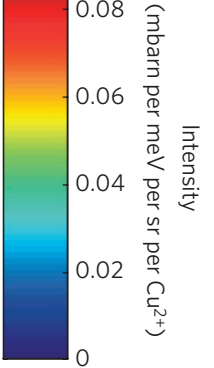

c

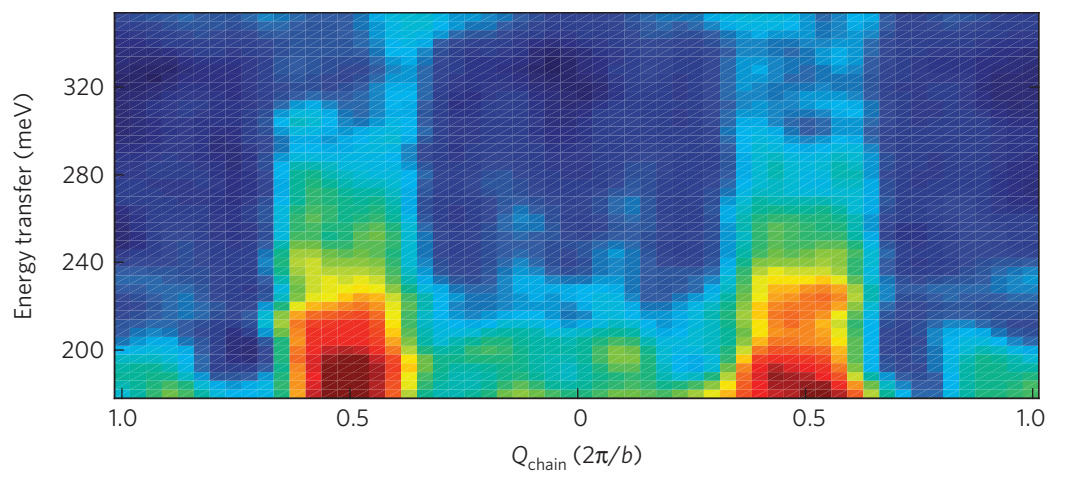

0.15

0.15

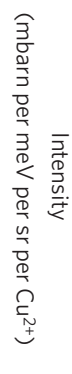

d

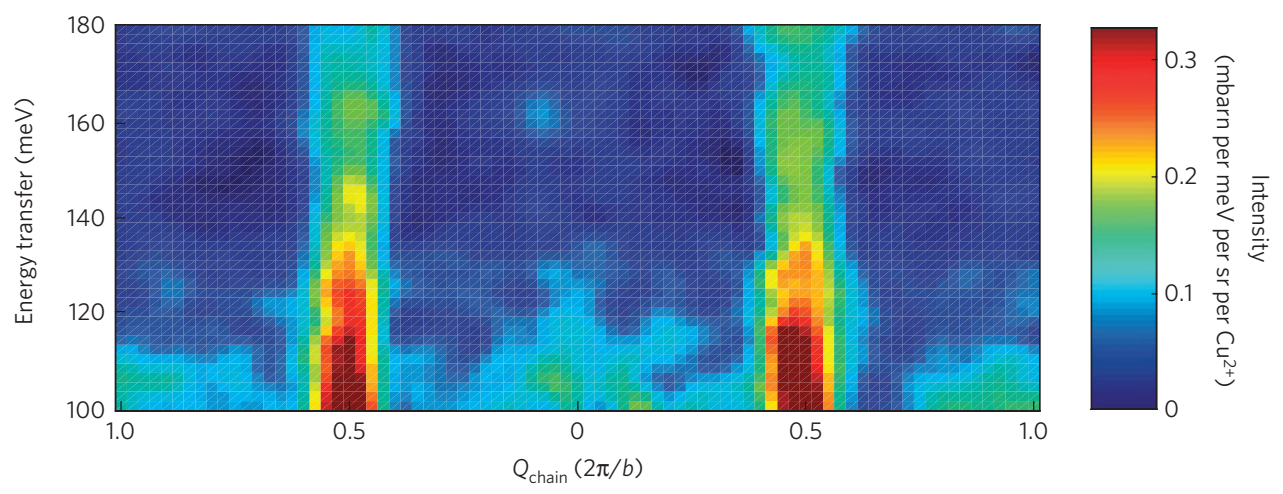

Figure 2 | Net magnetic INS intensity from $\mathrm{Sr}_{2} \mathrm{CuO}_{3}$ plotted as a function of momentum parallel to the chains and energy transfer. a-d, Optimization of resolution and intensity conditions required using four different incident neutron energies $E_{\mathrm{i}}$ : $1088 \mathrm{meV}$ (a), $794 \mathrm{meV}$ (b), $516 \mathrm{meV}$ (c) and $240 \mathrm{meV}$ (d). A choice of lower $E_{\mathrm{i}}$ results in superior momentum and energy resolution, but can measure excitations only up to $E_{\mathrm{i}}$. The data for each $E_{\mathrm{i}}$ were accumulated for one to several days. The intensity is in mbarn per steradian per millielectronvolt per $\mathrm{Cu}$ ion and the colour scale in each panel is adjusted to emphasize magnetic scattering.

are extremely small in $\mathrm{Sr}_{2} \mathrm{CuO}_{3}$, resulting in negligible interchain hopping and spin coupling and rendering this material the recordholder for one-dimensionality: 3D antiferromagnetism in $\mathrm{Sr}_{2} \mathrm{CuO}_{3}$ appears only below $T_{\mathrm{N}} \approx 5 \mathrm{~K}$ (ref. 27 ), which yields a factor $\geqslant 10^{3}$ smaller interchain exchange than the in-chain exchange. Magnetic orbitals and corresponding form factors used in our study are shown in Fig. 1b-h.

The colour contour maps in Fig. 2 show an overview of our INS data. They show the normalized intensity of magnetic scattering by the high-quality single crystal of $\mathrm{Sr}_{2} \mathrm{CuO}_{3}$ at 
a

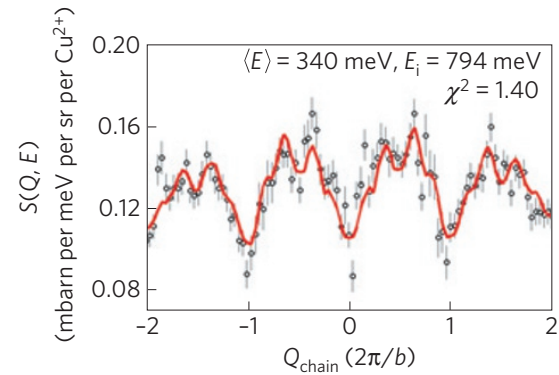

c

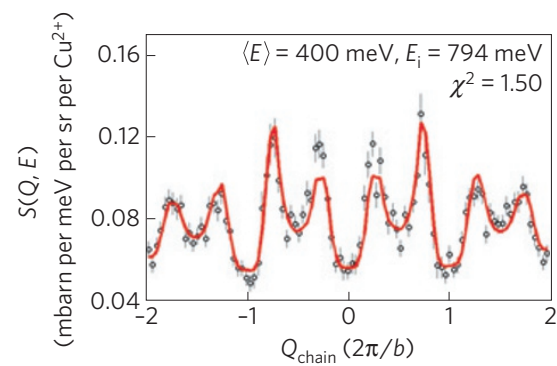

e

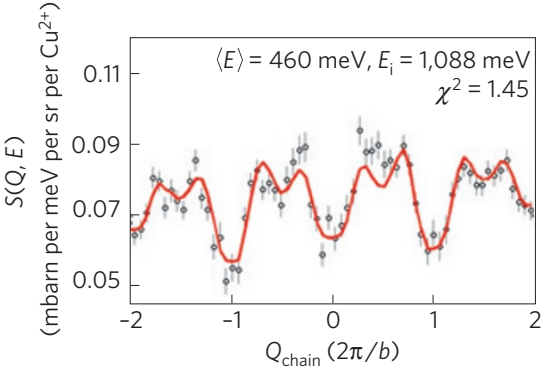

g

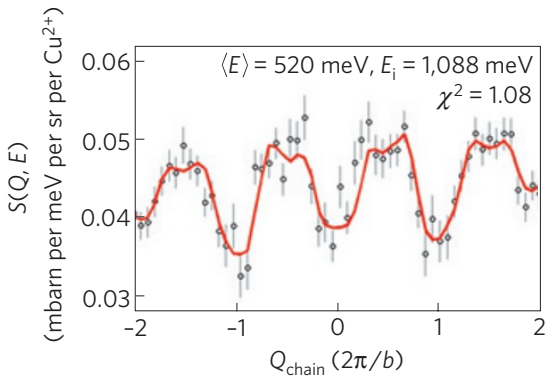

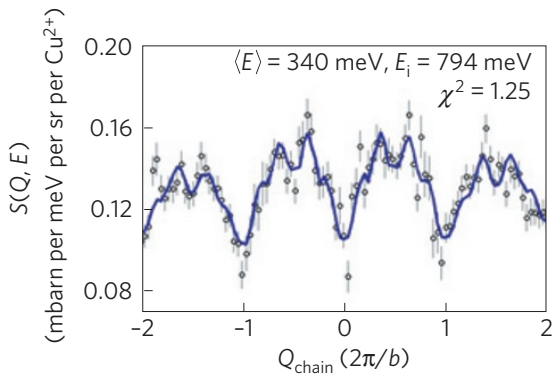

d

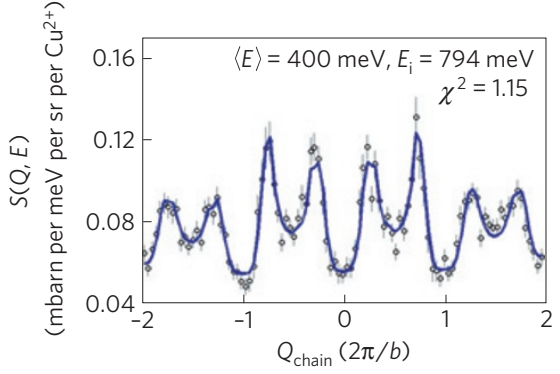

f

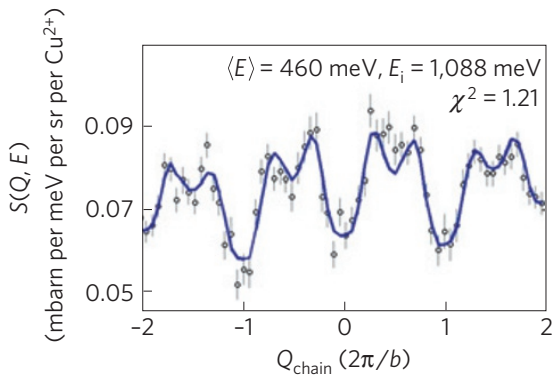

h

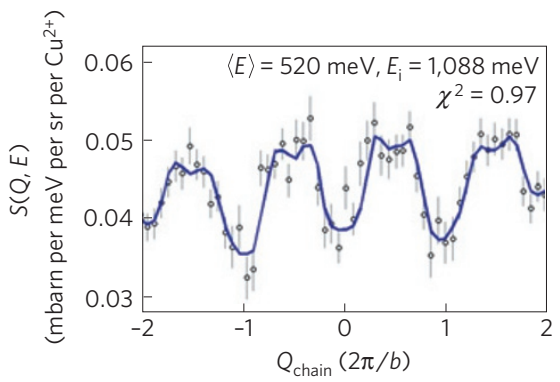

Figure 3 | Selected constant-energy cuts through the data as a function of momentum transfer parallel to $\mathbf{C u}-\mathbf{O}$ chains. a-h, The lines are fits to the exact two- and four-spinon scattering function for the spin-1/2 Heisenberg chain, using the ionic $\mathrm{Cu}^{2+}$ magnetic form factor of Fig. $1 d$ (a,c,e,g) and the LDA $+U$ covalent magnetic form factor of Fig. 1c (b,d,f,h). The error bars show the statistical error $\pm \sqrt{N}$ of the neutron count $N$. The in-chain exchange coupling and the intensity prefactor were the only parameters varied in these fits. Similar fits were also carried out for many other constant-energy cuts. The noticeably better agreement is achieved by using the covalent form factor. Its statistical significance is quantified by systematically lower $\chi^{2}$, which corresponds to the increase in the confidence level from $0.5,0.1$ and $0.2 \%$ for the fits in $\mathbf{a}, \mathbf{c}$ and e to $4.8,14.6$ and $7.6 \%$ for those in $\mathbf{b}, \mathbf{d}$ and $\mathbf{f}$, respectively.

$T \approx 5.5 \mathrm{~K}$. The measured non-magnetic signal-due to incoherent and multi-phonon scattering and so on-has in all cases been subtracted. The data cover two 1D Brillouin zones and were not symmetrized. Magnetic scattering corresponding to the multispinon triplet continuum ${ }^{25}$ emanating from $Q_{\text {chain }}= \pm 0.5$, where it is most intense, and extending to $E \sim 600 \mathrm{meV}$, is clearly seen repeated in both Brillouin zones. The continuum of excitations filling large regions of $\left(Q_{\text {chain }}, E\right)$ phase space is clearly visible in Fig. 2a,b. In Fig. 2c,d, it is possible to identify both strong spinon scattering emerging from $Q_{\text {chain }}= \pm 0.5$ and weaker returning spinon branches around $Q_{\text {chain }}=0, \pm 1$ with a $|\sin (\pi Q)|$-like dispersion extending up to $\approx 350 \mathrm{meV}$.

We analyse our data by fitting the $Q_{\text {chain }}$-dependent intensity of $1 \mathrm{D}$ constant-energy cuts at different energy transfers to the exact dynamical structure factor for the 1D spin-1/2 Heisenberg Hamiltonian including both the two-spinon and four-spinon contributions to $S\left(Q_{\text {chain }}, E\right)$ (ref. 25). Although higher-order excitations will also form a part of the excitation continuum, the calculated spectral weight arising from these two contributions is about $98 \%$ of the total, which is within our experimental error.

We began by fitting the measured intensities to the exact dynamical structure factor $S\left(Q_{\text {chain }}, E\right)$ (ref. 25) and using the anisotropic magnetic form factor of the $\mathrm{Cu}^{2+}$ ion, following the procedure adopted in previous studies ${ }^{6,13,28,29}$. Only two parameters are varied in such fitting: the in-chain exchange coupling $J$, which determines the overall energy scale of magnetic excitations, and the prefactor $A$, which accounts for the possible statistical and systematic errors of our intensity measurements and ideally should 

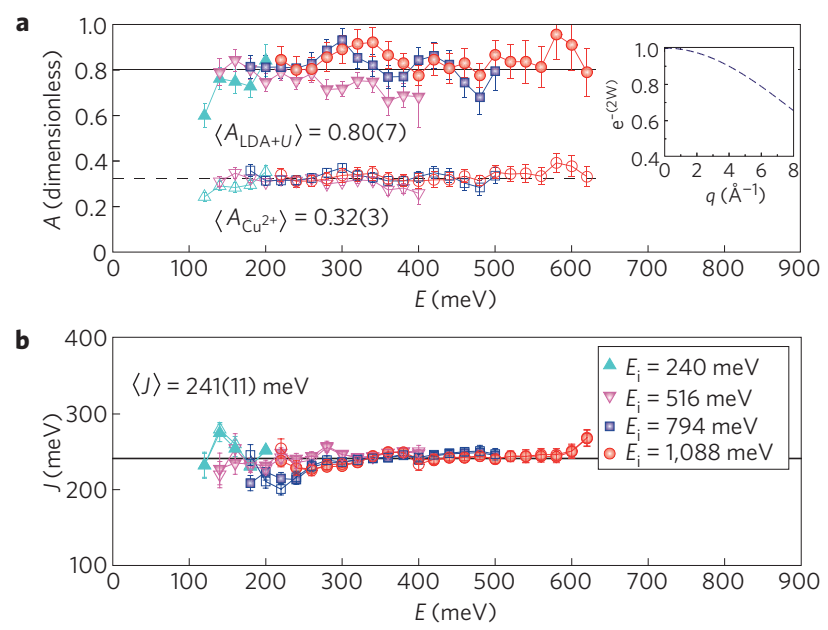

Figure 4 | The intensity prefactor $\boldsymbol{A}$ and the exchange integral $\boldsymbol{J}$.

$\mathbf{a}, \mathbf{b}$, Points were obtained by fitting a number of constant-energy scans collected with different incident energies, such as shown in Fig. 3, to the exact expression for the sum of two- and four-spinon scattering and using the LDA $+U$ covalent magnetic form factor (filled symbols) and that of the free $\mathrm{Cu}^{2+}$ ion (open symbols). The error bars show standard deviations derived from least-square fitting. The inset in a illustrates the magnitude of extra intensity suppression by the Debye-Waller factor, calculated for bulk copper ${ }^{21}$

be equal to one. Examples of the resulting fits are shown by solid lines in Fig. 3a,c,e,g, and the obtained parameters $A$ and $J$ are shown in Fig. 4. Although the overall agreement with the data in Fig. 3a,c,e,g is reasonable, the intensity prefactor $A=0.32(3)$ is far too small-it seems as if we are measuring only $1 / 3$ of the predicted intensity. This falls well beyond the most conservative estimates of statistical and systematic errors of our experiment, which are given by the error bars and the point scatter in Fig. 4, respectively. A similar factor of two to three missing intensity was reported in the related chain cuprate $\mathrm{SrCuO}_{2}$ - up to now presenting a puzzle ${ }^{13}$. As mentioned earlier, missing intensity was also reported in planar cuprates $^{5,6}$, although in the absence of an exact theory for $S(Q, E)$ in $2 \mathrm{D}$ it was difficult to quantify its significance.

Anticipating covalency as the prime suspect for these outstanding discrepancies, we computed the low-energy Wannier function and the corresponding magnetic form factor for $\mathrm{Sr}_{2} \mathrm{CuO}_{3}$ numerically using density functional theory with the LDA $+U$ functional, which has been successful in describing the magnetic insulating phase of the cuprates ${ }^{22-24}$. The results are shown in Fig. 1b,d and are compared in more detail with the ionic $\mathrm{Cu}^{2+}$ magnetic form factor in Fig. 1f-h. Figure $1 \mathrm{~b}$ clearly demonstrates strong hybridization between $\mathrm{Cu} d$ - and $\mathrm{O} p$-states and significant magnetic density on the oxygen sites. The resulting covalent magnetic form factor in Fig. 1d reflects this behaviour. A larger extent of the wavefunction in real space naturally leads to a smaller size of the form factor in wave-vector space and consequently smaller INS intensity at the same wave vector.

Fits of the data with the same dynamical structure factor but with the newly calculated covalent magnetic form factor of Fig. 1d are shown in Fig. 3b,d,f,h. The visibly better agreement of the new fits with the data is also quantified by the consistently lower values of the reduced $\chi^{2}\left(\chi^{2}=1.5\right.$ corresponds to $\approx 0.1 \%$ confidence level in the fit model, whereas $\chi^{2}$ near 1 -to $50 \%$ confidence, meaning that the model is as accurate as error bars on the data could afford). Most importantly, the value of the prefactor $A$ shown in Fig. 4 a now accounts for $\approx 80 \%$ of the expected intensity. This is a change by a factor $\approx 2.5$ - a marked correction to the ionic picture. The remaining small discrepancy of $20 \%$ is probably accounted for by the Debye-Waller factor, which results from the disorder of ionic positions in real materials and superposes a much weaker wave-vector-dependent reduction of the coherent scattering intensity. Its effect is illustrated in the inset of Fig. 4a. The values of the exchange coupling $J$ refined in both fits practically coincide, $J=241(11) \mathrm{meV}$, reflecting the fact that the excitation dispersion is sensitive to the positions of peaks in the measured intensities in $\left(Q_{\text {chain }}, E\right)$ space and not to their absolute values.

Thus, we find a marked suppression of the INS magnetic intensity in the antiferromagnetic cuprate $\mathrm{Sr}_{2} \mathrm{CuO}_{3}$, which results from the strong covalent bonding between the $\mathrm{Cu} 3 d$ orbitals and the $2 p$ orbitals of the surrounding $\mathrm{O}$ ligands. We were able to isolate and unambiguously identify this covalent magnetic INS reduction in the $1 \mathrm{D}$ case of $\mathrm{Sr}_{2} \mathrm{CuO}_{3}$ because it presents an exactly solvable spin system. However, this effect is common to all corner-sharing cuprates of the HTSC family and explains the missing magnetic INS intensity observed in these materials. In fact, we have also calculated the Wannier functions and the magnetic form factors for $\mathrm{La}_{2} \mathrm{CuO}_{4}$ and $\mathrm{SrCuO}_{2}$, and found very similar results. Therefore, an accurate account for the effects of covalency on the measured magnetic neutron intensities is absolutely required to obtain a quantitatively accurate experimental determination of the dynamical spin correlations and spininteraction energies in cuprates.

Surprisingly, previous neutron diffraction studies reported very little, if any covalent reduction of the ordered magnetic moment in cuprates $^{28-30}$. This controversy was pointed out by Kaplan et al. ${ }^{30}$, but the point was declared moot in view of the probably fortuitous agreement between the experiment and the semiclassical theory. Elastic neutron diffraction, however, probes $|F(\mathbf{Q})|^{2}$ only at a select number of points where magnetic Bragg peaks are present. Therefore, its sensitivity to covalency is very limited and is in fact constrained by the symmetry of the magnetic structure. In particular, in the case of the antiferromagnetic ordering found in $\mathrm{La}_{2} \mathrm{CuO}_{4}$, contributions of the covalent magnetic moments of the oxygens to magnetic Bragg peak intensities exactly cancel. INS, on the other hand, measures the magnetic form factor throughout $\mathbf{Q}$ space, wherever spin excitations are present. Hence, not only does magnetic INS probe spin fluctuations, it is established in our present study as a unique, non-perturbing experimental probe of wavefunctions occupied by the unpaired magnetic electrons. In cases where a reliable computation of spin correlations exists, magnetic INS could be used to investigate the Fourier transform of the magnetic electron density and thus probe directly the Wannier functions of the unpaired electrons.

\section{Methods}

The success of our study rested on an outstanding progress in experimental and theoretical techniques, which has occurred over the past decade. It combined (1) availability of large, high-quality crystals, (2) recent development of time-of-flight neutron scattering instrumentation, (3) exact Bethe ansatz results for $S(Q, E)$ and (4) recent advances in the first-principles calculations, including construction of the low-energy Wannier functions.

The $\mathrm{Sr}_{2} \mathrm{CuO}_{3}$ sample used in our measurements was composed of three large single crystals with a combined mass of $18.45 \mathrm{~g}$. All crystals were grown using the travelling solvent-floating zone method and had a mosaic equal to or smaller than $0.3^{\circ}$ full-width at half-maximum. The crystals were mutually coaligned on an aluminium sample holder to better than $0.4^{\circ}$ and $0.9^{\circ}$ full-width at half-maximum with respect to rotations around the $a$ and $c$ lattice directions, respectively. The sample was mounted on the cold head of the closed-cycle refrigerator in the evacuated scattering chamber of the MAPS spectrometer at the ISIS spallation neutron source at the Rutherford Appleton Laboratory, UK. The $b-c$ plane was nearly horizontal and the $b$ (chain) direction was aligned perpendicular to the incident neutron beam.

In direct-geometry time-of-flight neutron spectrometers such as MAPS, a pulse of monochromatic neutrons with energy $E_{\mathrm{i}}$ is incident on the sample and then the energy lost or gained by the neutron in the sample is ascertained by measuring the time taken for the neutrons to travel to the detector. By using time-resolved detectors, the whole energy-transfer range $E<E_{\mathrm{i}}$ is measured in a single pulse. The INS spectrum of $\mathrm{Sr}_{2} \mathrm{CuO}_{3}$ was measured at four different incident neutron 
energies, 240, 516, 794 and 1,088 $\mathrm{meV}$, and with the Fermi chopper spinning at 400, 400, 500 and $600 \mathrm{~Hz}$, respectively. Although the lower incident energies do no allow the full energy range of the spinon excitations to be measured, they provide better energy resolution on the lower energy excitations. Data were normalized by measuring the incoherent neutron cross-section of a vanadium sample of known mass in each configuration. Each cut shows data obtained by properly adding the measured intensity within several Brillouin zones in the direction perpendicular to chains. The non-magnetic intensity arising from the incoherent and coherent multiple phonon and multi-phonon scattering by the sample and its environment was fitted to a simple smoothly varying functional form $A+B \Phi^{2}+C Q_{\text {chain }}$, where $\Phi$ is the angle by which neutrons are scattered, and that allows for a small empirically observed component $\sim Q_{\text {chain }}$, accounting for slight asymmetry of the beam-defining aperture. The cuts in Fig. 3 show unsubtracted data; Fig. 2 has had the fitted background subtracted. The fits of $S(\mathbf{Q}, E)$ to the data were carried out using the TовҮғгт program (T.G.P., unpublished), which uses Monte Carlo integration to convolute the instrumental resolution function with models for the scattering cross-section.

The theoretical $a b$ initio electronic structure calculation was carried out with $\mathrm{LDA}+U(U=8 \mathrm{eV})$ approximation of density functional theory implemented in the WIEN2k code using the full-potential, all-electron, linearized augmented plane-wave basis. In $\mathrm{Sr}_{2} \mathrm{CuO}_{3}$, the $d$ shell has a single hole in the $x^{2}-y^{2}$ orbital. The Wannier function is constructed from LDA $+U$ orbitals, such that it preserves the local point-group symmetry and spans the low-energy Hilbert space of the hole in the electronic structure $e^{22,23}$. This describes the magnetic electron density, correctly accounting for the hybridization with surrounding $p$ orbitals of the oxygen ligands. Within a few per cent accuracy, the obtained Wannier function can be approximated by the following linear combination of the atomic-like orbitals within the $\mathrm{CuO}_{4}$ plaquette ${ }^{20}$ $0.8\left|\mathrm{~d}_{x^{2}-y^{2}}, \mathrm{Cu}\right\rangle+0.28\left(\left|\mathrm{p}_{x}, \mathrm{O}(1)\right\rangle-\left|\mathrm{p}_{x}, \mathrm{O}(2)\right\rangle\right)+0.3\left(\left|\mathrm{p}_{y}, \mathrm{O}(3)\right\rangle-\left|\mathrm{p}_{y}, \mathrm{O}(4)\right\rangle\right)$. The magnetic form factor is the Fourier transform of the norm squared of this low-energy Wannier function.

\section{Received 21 April 2009; accepted 26 August 2009;} published online 4 October 2009

\section{References}

1. Eschrig, M. The effect of collective spin-1 excitations on electronic spectra in high- $T_{\mathrm{c}}$ superconductors. Adv. Phys. 55, 47-183 (2006).

2. Tranquada, J. M. et al. Quantum magnetic excitations from stripes in copper oxide superconductors. Nature 429, 534-538 (2004).

3. Hayden, S. M., Mook, H. A., Dai, P., Perring, T. G. \& Doğan, F. The structure of the high-energy spin excitations in a high-transition-temperature superconductor. Nature 429, 531-534 (2004).

4. Fong, H. F. et al. Neutron scattering from magnetic excitations in $\mathrm{Bi}_{2} \mathrm{Sr}_{2} \mathrm{CaCu}_{2} \mathrm{O}_{8+\delta}$. Nature 398, 588-591 (1999).

5. Bourges, P., Casalta, H., Ivanov, A. S. \& Petitgrand, D. Superexchange coupling and spin susceptibility spectral weight in undoped monolayer cuprates. Phys. Rev. Lett. 79, 4906-4909 (1997).

6. Coldea, R. et al. Spin waves and electron interactions in $\mathrm{La}_{2} \mathrm{CuO}_{4}$. Phys. Rev. Lett. 86, 5377-5380 (2001).

7. Dahm, T. et al. Strength of the spin-fluctuation-mediated pairing interaction in a high-temperature superconductor. Nature Phys. 5, 217-221 (2009).

8. Hinkov, V. et al. Spin dynamics in the pseudogap state of a high-temperature superconductor. Nature Phys. 3, 780-785 (2007).

9. Woo, H. et al. Magnetic energy change available to superconducting condensation in optimally doped $\mathrm{YBa}_{2} \mathrm{Cu}_{3} \mathrm{O}_{6.95}$. Nature Phys. 396, 600-604 (2006).

10. Demler, E. \& Zhang, F.-C. Quantitative test of a microscopic mechanism of high-temperature superconductivity. Nature 396, 733-735 (1998).

11. Kee, H.-Y., Kivelson, S. A. \& Aeppli, G. Spin-1 neutron resonance peak cannot account for electronic anomalies in the cuprate superconductors. Phys. Rev. Lett. 88, 257002 (2002).
12. Lorenzana, J., Seibold, G. \& Coldea, R. Sum rules and missing spectral weight in magnetic neutron scattering in the cuprates. Phys. Rev. B 72, 224511 (2005).

13. Zaliznyak, I. A. et al. Spinons in the strongly correlated copper oxide chains in $\mathrm{SrCuO}_{2}$. Phys. Rev. Lett. 93, 087202 (2004).

14. Anderson, P. W. The resonating valence bond state in $\mathrm{La}_{2} \mathrm{CuO}_{4}$ and superconductivity. Science 235, 1196-1198 (1987).

15. Anderson, P. W. New approach to the theory of superexchange interaction. Phys. Rev. 79, 2-13 (1959).

16. Pauling, L. The nature of the chemical bond. Application of results obtained from the quantum mechanics and from a theory of paramagnetic susceptibility to the structure of molecules. J. Am. Chem. Soc. 53, 1367-1400 (1931).

17. Alperin, H. A. Aspherical 3d electron distribution in $\mathrm{NI}^{++}$. Phys. Rev. Lett. 6, 55-57 (1961)

18. Hubbard, J. \& Marshall, W. Covalency effects in neutron diffraction from ferromagnetic and antiferromagnetic salts. Proc. Phys. Soc. 86, 561-572 (1965).

19. Plakhty, V. P., Gukasov, A. G., Papoular, R. J. \& Smirnov, O. P. Spin density on ligands $\mathrm{O}^{2-}$ and covalency of $\mathrm{Fe}^{3+}$ ions in octahedral sites of the $\mathrm{Ca}_{3} \mathrm{Fe}_{2} \mathrm{Ge}_{3} \mathrm{O}_{12}$ garnet: A polarised neutron diffraction study. Europhys. Lett. 48, 233-239 (1999).

20. Zhang, F. C. \& Rice, T. M. Effective Hamiltonian for the superconducting $\mathrm{Cu}$ oxides. Phys. Rev B 37, 3759-3762 (1988).

21. Shirane, G., Shapiro, S. M. \& Tranquada, J. M. Neutron Scattering with a Triple Axis Spectrometer 35-54, 126 (Cambridge Univ. Press, 2002).

22. Pickett, W. E. Electronic structure of the high-Tc cuprates. Rev. Mod. Phys. 61, 433-512 (1989).

23. Ku, W., Rosner, H., Pickett, W. E. \& Scalettar, R. T. Insulating ferromagnetism in $\mathrm{La}_{4} \mathrm{Ba}_{2} \mathrm{Cu}_{2} \mathrm{O}_{10}$ : An ab initio Wannier function analysis. Phys. Rev. Lett. 89, 167204 (2002).

24. Neudert, R. et al. Four-band extended Hubbard Hamiltonian for the one-dimensional cuprate $\mathrm{Sr}_{2} \mathrm{CuO}_{3}$ : Distribution of oxygen holes and its relation to strong intersite Coulomb interaction. Phys. Rev. B 62, 10752-10765 (2000).

25. Caux, J.-S. \& Hagemans, R. The four-spinon dynamical structure factor of the Heisenberg chain. J. Stat. Mech. P12013 (2006).

26. Suzuura, H., Yasuhara, H., Furusaki, A., Nagaosa, N. \& Tokura, Y. Singularities in optical spectra of quantum spin chains. Phys. Rev. Lett. 76, 2579-2582 (1996).

27. Kojima, K. M. et al. Reduction of ordered moment and Néel temperature of quasi-one-dimensional antiferromagnets $\mathrm{Sr}_{2} \mathrm{CuO}_{3}$ and $\mathrm{Ca}_{2} \mathrm{CuO}_{3}$. Phys. Rev. Lett. 78, 1787-1790 (1997).

28. Fretfolt, T., Shirane, G., Mitsuda, S., Remeika, J. P. \& Cooper, A. S. Magnetic form factor in $\mathrm{La}_{2} \mathrm{CuO}_{4}$. Phys. Rev. B 37, 137-142 (1988).

29. Shamoto, S., Sato, M., Tranquada, J. M., Sternlieb, B. J. \& Shirane, G. Neutron-scattering study of antiferromagnetism in $\mathrm{YBa}_{2} \mathrm{Cu}_{3} \mathrm{O}_{6.15}$. Phys. Rev. $B$ 48, 13817-13825 (1993).

30. Kaplan, T. A., Mahanti, S. D. \& Chang, H. Spin fluctuations and covalence in the half-filled narrow-band Hubbard model. Phys. Rev. B 45, R2565-R2568 (1992).

\section{Acknowledgements}

We acknowledge discussions with J. Tranquada, F. Essler, H. Benthien and D. F. McMorrow. Work at BNL was supported by the Office of Science, US Department of Energy under Contract No. DE-AC02-98CH10886. J.-S.C. acknowledges support from the FOM foundation.

\section{Author contributions}

Project planning: I.A.Z., T.G.P.; sample preparation: G.D.G.; experiments: I.A.Z., T.G.P., A.C.W., A.T.S.; theory: J.S.C., C.C.L., W.K.; data analysis: A.C.W., T.G.P., I.A.Z.; paper writing: I.A.Z., A.C.W.

\section{Additional information}

Reprints and permissions information is available online at http://npg.nature.com/ reprintsandpermissions. Correspondence and requests for materials should be addressed to I.A.Z. 